S.M. CHOI

KODAI MATH. J.

16 (1993), 332-333

\title{
CORRECTION TO : 3-DIMENSIONAL SPACE-LIKE SUBMANIFOLDS WITH PARALLEL MEAN CURVATURE VECTOR OF AN INDEFINITE SPACE FORM
}

\author{
By SOON MEEN CHOI
}

In Theorem 2 in [1], we considered a 3-dimensional complete space-like submanifold with non-zero parallel mean curvature vector $\boldsymbol{h}$ of $M_{p}^{n+p}(c)$ which satisfied

$$
\sup \operatorname{Ric}(M)<\frac{3}{2}(p-3)\left(H^{2}-c\right)
$$

and

$$
S \geqq 3 p H^{2}-3(p-1) c .
$$

At that time, we asserted the possibility of the existence of some non-trivial examples for this situation. However, we had some mistakes in the argument. These occured in the computation written on the bottom of page 293 and the top of page 294 in [1]. These should be corrected as follows:

$$
n^{2} H^{2}=n^{2} c-\sum_{r=1}^{q+1} n_{r}^{2} c_{r}, \quad S=n c-\sum_{r=1}^{q+1} n_{r} c_{r} .
$$

In fact, Theorem 2 in [1] should be replaced by the following

THEOREM 2. Let $M$ be a 3-dimensional complete space-like submanifold with non-zero parallel mean curvature vector of $M_{p}^{n+p}(c)$. If it satisfies (1) and

$$
S=3 p H^{2}-3(p-1) c,
$$

then $p=1, c>0$ and $M$ is congruent to a totally umbilic hypersurface $\boldsymbol{S}^{\mathbf{s}}\left(c_{1}\right)$ in a de-Sitter space $\boldsymbol{S}_{\mathbf{1}}^{4}(c)\left(c_{1}=c-H^{2}\right)$.

Proof. In order to prove Theorem 2, the following fact is needed.

Proposition. Let $M$ be an $n$-dimensional complete space-like submanifold

Received February 5, 1993. 
with non-zero parallel mean curvature vector of $M_{p}^{n+p}(c)$. If $M$ is a pseudoumbilical submanifold, then $M$ is a maximal submanifold of a totally umbilical hypersurface $M_{p-1}^{n+p-1}(\bar{c})$ of $M_{p}^{n+p}(c)$.

The generalized case of Proposition was proved by Chen in [2].

Now, let us prove Theorem 2 .

When $p=1$, it follows immediately from Theorem in [1] that $c>0$ and $M$ is congruent to $\boldsymbol{S}^{3}\left(c_{1}\right)$ in $\boldsymbol{S}_{1}^{4}(c)$.

Next, we suppose that $p \geqq 2$. In the section 5 in [1], we proved that $M$ must be pseudo-umbilic under the given conditions. By means of Proposition, $M$ is to be a maximal submanifold of a totally umbilic hypersurface $M_{p-1}^{s+p-1}\left(c^{\prime}\right)$ of $M_{p}^{3+p}(c)$. If $c^{\prime} \geqq 0$, from Ishihara's theorem [3], then $M$ is totally geodesic in $M_{p-1}^{3+p-1}\left(c^{\prime}\right)$. That is, $M$ is totally umbilic in $M_{p}^{3+p}(c)$. It follows from (3) that $H^{2}=c$, that is, $c^{\prime}=0$. This means that Ricci curvature of $M$ is zero. These are contradiction to the condition (1). Hence the case $c^{\prime} \geqq 0$ can not be occured. Then we may consider the case $c^{\prime}<0$. Let $S^{\prime}$ be a squared norm of the second fundamental form of $M$ in $M_{p-1}^{3+p-1}\left(c^{\prime}\right)$. From (3), we have $S^{\prime}=$ $-3(p-1) c^{\prime}$. So, by means of Ishihara's theorem [3], $M$ is obtained to be congruent to

$$
\begin{aligned}
& \boldsymbol{H}^{2}\left(c_{1}\right) \times \boldsymbol{H}^{1}\left(c_{2}\right), \quad p=2, \\
& \boldsymbol{H}^{1}\left(c_{1}\right) \times \boldsymbol{H}^{1}\left(c_{2}\right) \times \boldsymbol{H}^{1}\left(c_{3}\right), \quad p=3 .
\end{aligned}
$$

The Ricci curvatures of (5) and (6) do not satisfy the condition (1). Therefore, in the case $p \geqq 2$, there is no existence of $M$ satisfing the given conditions. Thus, we obtain the conclusion of the theorem.

\section{REFERENCES}

[1] S.M. C $\mathrm{HOI}$, 3-dimensional space-like submanifold with parallel mean curvature vector of an indefinite space form, Kodai Math. J. 15 (1992), 279-295.

[2] B.Y. CHEN, Finite type submanifolds in pseudo-Euclidian space and applications, Kodai Math. J. 8 (1985), 358-374.

[3] T. IsHiHARA, Maximal spacelike submanifolds of a pseudoRiemannian space of constant curvature, Michigan Math. J. 35 (1988), 345-352.

INSTitute of Mathematics,

UNIVERSITY OF TSUKUBA,

305 IBARAKI, JAPAN 
